\title{
Parenting self-efficacy, parenting stress and child behaviour before and after a parenting programme
}

\author{
Linda Bloomfield ${ }^{1}$ and Sally KendalI ${ }^{2}$ \\ ${ }^{1}$ Research Fellow, Centre for Research in Primary and Community Care, University of Hertfordshire, Hatfield, UK \\ ${ }^{2}$ Director, Centre for Research in Primary and Community Care, University of Hertfordshire, Hatfield, UK
}

\begin{abstract}
Aim: To explore whether changes in parenting self-efficacy after attending a parenting programme are related to changes in parenting stress and child behaviour. Background: Adverse parenting is a risk factor in the development of a range of health and behavioural problems in childhood and is predictive of poor adult outcomes. Strategies for supporting parents are recognised as an effective way to improve the health, well-being and development of children. Parenting is influenced by many factors including the behaviour and characteristics of the child, the health and psychological well-being of the parent and the contextual influences of stress and support. Parenting difficulties are a major source of stress for parents, and parenting self-efficacy has been shown to be an important buffer against parenting stress. Methods: In all, 63 parents who had a child under the age of 10 years took part in the research. Of those, 58 returned completed measures of parenting self-efficacy, parenting stress and child behaviour at the start of a parenting programme and 37 at three-month followup. Findings: Improvements in parenting self-efficacy and parenting stress were found at follow-up, but there was less evidence for improvements in child behaviour. The findings clearly suggest a relationship between parenting self-efficacy and parenting stress; parents who are feeling less efficacious experience higher levels of stress, whereas greater parenting self-efficacy is related to less stress. This study adds to the evidence that parent outcomes may be a more reliable measure of programme effectiveness than child outcomes at least in the short term.
\end{abstract}

Key words: child behaviour; parenting programmes; parenting self-efficacy; parenting stress; TOPSE

Received 20 July 2011; revised 12 October 2011; accepted 31 January 2012;

first published online 2 April 2012

\section{Background}

There is increasing recognition and concern that strategies for supporting parents are the most effective way to improve the health, well-being and development of children, and that adverse parenting is a risk factor for the development of a

Correspondence to: Linda Bloomfield, Centre for Research in Primary and Community Care, University of Hertfordshire, College Lane, Hatfield, Hertfordshire AL10 9AB, UK. Email:

1.j.bloomfield@herts.ac.uk

(C) Cambridge University Press 2012 range of health problems both in childhood and adulthood (World Health Organization, 2002; Her Majesty's Government, 2006; Stewart-Brown, 2008). The development of the Healthy Child Programme (Department of Health, 2009) is based on evidence of early intervention by health practitioners that will promote child development in the early years. Much of the success of this programme will depend on health visitors and public health nurses providing support and information for parents (Department of Health, 2011). Failure to address children's needs has been linked to 
negative outcomes in terms of later social and emotional development (Macdonald, 2001).

Behaviour problems in children are an important area in which parenting has an impact. Parenting is recognised as being key to the prevention of crime and educational failure (Stewart-Brown, 2008) and there is a growing body of international evidence that parenting programmes can be both effective and cost-effective in helping children with challenging behaviour (Scott et al., 2001a; Gardner et al., 2006; Petrie et al., 2007; Edwards et al., 2007). Support for parents contributes to the prevention of longer term problems of neglect, abuse and antisocial behaviour, and this is endorsed by Allen's 'campaign on parenting' (Allen, 2011). The increasing prevalence of child behaviour problems and their association with parenting have led to the development of a range of interventions by statutory and voluntary agencies that focus on improving family relationships and reducing behavioural problems (Scott, 1998; Anderson et al., 2005). Group parenting programmes have shown positive results with parents of children with clinically defined behaviour disorders and those at high risk of developing behaviour problems (Scott and Stradling, 1987; Barlow and Stewart-Brown, 2000; Scott et al., 2001b).

Parental functioning is determined by the interplay of characteristics of the child, the personal and psychological resources of the parent, and the contextual sources of stress and support (Belsky, 1984). Belsky proposed that well-functioning parenting can be largely explained and understood in relation to these three key determinants in the process of parenting. Both child temperament (Putnam et al., 2002) and parental psychopathology (Goodman and Gotlib, 2002) contribute to parenting behaviour and the pattern of interaction between parent and child.

Perceived parenting difficulties is a major source of stress for parents (Vondra and Belsky, 1993), particularly when the child is viewed as moody or demanding and interactions between parent and child are perceived by the parent as difficult (Ostberg and Hagekull, 2000). Parenting stress arises from the parent's perception of their own competence in the parenting role, as well as a perception of their child's behaviour (Abidin and Burke, 1978; Abidin, 1997). Parenting stress is thought to involve characteristics of the child, the parent and the context (Abidin, 1986; Ostberg and Hagekull, 2000) and has been linked to both parenting behaviour and child functioning (Deater-Deckard, 1998). Parenting stress acts to negatively influence parenting behaviour (Abidin, 1986) and negative parenting behaviour has been linked to higher parental stress and more problem behaviours in children (Deater-Deckard and Scarr, 1996).

Parenting self-efficacy has been shown to be an important buffer against parenting stress (Coleman and Karraker, 1998; Raikes and Thompson, 2005). Grounded in social cognitive theory (Bandura, 1989; 1997), parenting self-efficacy is broadly defined as an individual's appraisal of his or her competence in the parenting role (Coleman and Karraker, 2000; Kendall and Bloomfield, 2005). Greater perceived competence in parenting is associated with the tendency to assess situations as less problematic and to feel confident that difficulties can be resolved (Mash and Johnston, 1990; Coleman and Karraker, 1998; Coleman and Karraker, 2003). A key tenet of self-efficacy theory is that a person's self-efficacy expectations in any domain of behaviour will be developed by performance mastery and vicarious experience and learning through role modelling (Bandura, 1982; 1986; 1989). Rather than a fixed personality trait, self-efficacy is a dynamic and emerging process that is modified by task and situational demands, as well as changing individual factors (Sevigny and Loutzenhiser, 2009). Parenting selfefficacy has been identified as a major determinant of parenting behaviours and closely linked to child development outcomes and psychosocial child adjustment (Teti and Gelfand, 1991; Gross and Tucker, 1994; Coleman and Karraker, 2003; Jones and Prinz, 2005).

Given previous research on parenting, the theoretical contribution of parenting self-efficacy to parenting stress seems highly likely. It is known from previous studies in the United Kingdom that parenting self-efficacy improves after attending a range of community-based parenting programmes (Bloomfield and Kendall, 2007; 2010). Parenting programmes provide opportunities for parents to develop their self-efficacy through learning and achieving positive behaviours, by experiencing other parents' success, and through encouragement from programme facilitators and other parents (Kendall, 1991; Bloomfield and Kendall, 2007). Recent parenting programme evaluations have demonstrated increased parenting self-efficacy after attending a parenting programme and this has 
shown to be sustained over time (Bloomfield and Kendall, 2007; 2010). Parents felt more confident in all domains of their parenting role and had positive expectations of the effect on their child at the end of the programme and four months afterwards.

With Belsky's model of the determinants of parenting in mind, we wanted to further the research on parenting programme outcomes. We selected a before and after quasi-experimental design without randomisation and measured parenting self-efficacy, parenting stress and child behaviour at baseline and three months following the end of the parenting programme. We aimed to explore whether changes in parenting self-efficacy after attending a parenting programme are related to changes in parenting stress and child behaviour.

\section{Methods}

\section{Intervention}

Parenting support is provided through a range of group-based programmes that have various approaches to supporting parents to become more effective and confident. '123Magic' (Phelan, 2004) is a parenting programme that encourages parents of children between the ages of 2 and 12 to explore, discuss and practise positive parenting strategies before taking them home to their families. The programme consists of six weekly sessions each of $2 \mathrm{~h}$, delivered by trained parenting programme facilitators. '123Magic' has been rated highly on research evidence (Bradley et al., 2003) using the California Evidence-Based Clearinghouse for Child Welfare (CEBC) scientific rating scale and also on the child welfare scale. A modified version of '123Magic' was the programme of choice for the current study as it was widely used in the area where the study took place and has demonstrated an impact on parenting self-efficacy (Bloomfield and Kendall, 2010). Through group work, parents have the opportunity to listen to other parents and share their parenting experiences. Parents can tailor approaches to their own family circumstances and gain support and validation for their individual techniques.

\section{Measures}

Tool to measure parenting self-efficacy (TOPSE)

TOPSE is a parenting programme evaluation tool that is sensitive and specific to parenting in the United Kingdom and takes into account the views and experiences of parents from a diverse range of cultural, educational and social backgrounds (Bloomfield et al., 2005; Kendall and Bloomfield, 2005). The theoretical underpinning of TOPSE is based on the self-efficacy theory developed by Albert Bandura (Bandura, 1982; 1986; 1989). TOPSE is a multi-dimensional instrument of 48 statements within eight scales, each scale having six statements and representing a distinct dimension of parenting: emotion and affection, play and enjoyment, empathy and understanding, control, discipline and boundaries, pressures, self-acceptance, learning and knowledge. The items are rated on an 11-point Likert scale where 0 represents completely disagree and 10 represents completely agree. The scale contains positive and negatively worded items and the responses are summed to create a total score; the lower the score, the lower the level of parenting self-efficacy.

\section{The parenting stress index (PSI) short form}

The PSI (Abidin, 1986) was developed as a screening and diagnostic instrument for use with parents of children aged 12 and below, with the primary focus being the pre-school child. The PSI identifies parent-child systems under stress and at risk for dysfunctional parenting and the development of emotional pathology in children. The PSI short form is a direct derivative of the full-length test and consists of 36 items to yield a total stress score from three scales to measure parental distress, dysfunctional interaction and difficult child.

The strength and difficulties questionnaire (SDQ)

The SDQ (Goodman, 1997) is a 25-item behavioural screening questionnaire to measure five distinct domains of child behaviour in children aged 3 to 16 . The 25 items are divided between five scales of five items each, generating scores for conduct problems, hyperactivity-inattention, emotional symptoms, peer problems and prosocial behaviour, and is completed by parents or teachers. The SDQ discriminates between children with clinically significant behaviour problems, borderline problems and those with no problems, and it focuses on strengths and difficulties. 


\section{Demographic questionnaire}

This was collected at baseline and included variables to measure family structure, ethnicity, working status, parental age and parity. It enabled a comparison of parents who completed both sets of questionnaires with those who dropped out of the study.

\section{Participants}

All parents of children from six months to ten years who were attending '123Magic' parenting programmes in the county where the study took place were eligible to take part. Fourteen programmes running between January 2009 and June 2009 were included. Only parents who attended all six sessions of the parenting programme were included. These programmes were run in a number of Children's Centres by trained parenting programme facilitators. Each programme was run by two facilitators from a range of backgrounds, including health visitors, family centre workers and parents. All facilitators had undertaken certified courses in group dynamics and handling sensitive issues in groups, as well as training in the specific parenting programme. Parents generally self-referred onto the programme or were recommended.

\section{Data collection}

The parenting programme was provided in a community setting, over six weeks of 2 hours at each session. All facilitators had the same training in delivering the parenting programme. Data were collected over an eight-month period. A member of the research team attended the first session of each parenting programme to talk to parents about the study and respond to questions and concerns. A study information sheet and consent form was given to parents to take home. Parents were asked to sign and return the consent form the following week if they agreed to take part.

Parents who provided signed consent completed baseline questionnaires of all measures at the start of the second session. These were collected by the programme facilitator and delivered to the research team. Three months following the end of the programme, parents were sent a further copy of each questionnaire for completion and returned to the research team in prepaid addressed envelopes. Questionnaires were coded with unique participant identity numbers to ensure that all sets of questionnaires were matched to the participant.

\section{Ethical considerations}

Approval for the study was granted by the University Research Ethics Committee. Parents attending parenting programmes were given an information sheet outlining the study, together with a consent form to complete and return to the parenting programme facilitator.

\section{Data analysis}

The data were analysed using the Statistical Package for the Social Sciences (SPSS) version 14.0. Paired $t$-tests were conducted to determine differences in scores from baseline to threemonth follow-up on measures of TOPSE, PSI and SDQ. Kruskall-Wallis independent samples tests were conducted on all measures to test whether there was a difference in baseline scores between parents who completed both sets of questionnaires and those who dropped out of the study. SPSS correlation methods were conducted to look at the relationship between TOPSE with both PSI and SDQ scores.

\section{Estimates of reliability and validity}

Previous studies have provided support for the reliability and validity of TOPSE. For the current study, internal consistency reliability for each instrument was estimated at baseline through the use of Cronbach's alpha coefficients (Table 1).

\section{Results}

Data were collected for 63 parents attending 14 parenting programmes. All parents completed the SDQ and PSI baseline questionnaires, including 57 mothers $(90.5 \%)$ and 6 fathers. Fifty-eight of those parents completed all baseline TOPSE scales, including 52 mothers and 6 fathers. The age range, $n=63$, was from 23 to 57 years (mean age 37.4 years). Fifty-seven parents lived with their spouse or partner and the remaining six parents $(9.5 \%)$ lived alone. All parents had between one and five children, the majority (36) having two children and three parents had adopted children. Thirty-nine parents $(60 \%)$ were working either full or part time, $30(48 \%)$ had left full-time education by the 
Table 1 Pre-programme Cronbach's $\alpha$ reliability coefficients for all scales

\begin{tabular}{lll}
\hline Scale & $n$ & $\alpha$ \\
\hline TOPSE 1: Emotion \& Affection & 58 & 0.775 \\
TOPSE 2: Play \& Enjoyment & 58 & 0.904 \\
TOPSE 3: Empathy \& Understanding & 58 & 0.898 \\
TOPSE 4: Control & 58 & 0.857 \\
TOPSE 5: Discipline \& Setting Boundary & 58 & 0.852 \\
TOPSE 6: Pressure & 58 & 0.778 \\
TOPSE 7: Self-acceptance & 58 & 0.904 \\
TOPSE 8: Learning \& Knowledge & 58 & 0.821 \\
Total TOPSE (sum of TOPSE 1-8) & & 0.914 \\
& & \\
PSI: Parental Distress (PD) & 63 & 0.882 \\
PSI: Parent-Child Dysfunctional Interaction & 63 & 0.897 \\
(P-CDI) & & \\
PSI: Difficult Child (DC) & 63 & 0.889 \\
Total PSI Stress (sum of PD, P-CDI \& DC) & & 0.858 \\
SDQ: Emotional Symptoms & 63 & 0.753 \\
SDQ: Conduct Problems & 63 & 0.715 \\
SDQ: Hyperactivity & 63 & 0.835 \\
SDQ: Peer Problems & 63 & 0.634 \\
SDQ: Prosocial & 63 & 0.737 \\
Total Difficulties Score (not prosocial) & & 0.687 \\
\hline TOPSE & &
\end{tabular}

TOPSE = tool to measure parenting self-efficacy; $\mathrm{PSI}=$ parenting stress index; $\mathrm{SDQ}=$ strength and difficulties questionnaire.

age of 16 years, a further $27(43 \%)$ continued to 18 years and five $(8 \%)$ attended higher education. Fifty-eight parents were of White British ethnicity.

Data were collected for 37 parents $(59 \%)$ at three-month follow-up, including 31 mothers and 6 fathers. All parents returned the completed TOPSE, SDQ and PSI questionnaires, including 31 mothers and 6 fathers. The age range of parents completing follow-up questionnaires was 23-50 years (mean age 37.7 years), and 36 were living with their spouse or partner and one parent lived alone. Parents had between one and three children and three had adopted children. Twentythree parents $(62 \%)$ were working full or part time. Thirty-six per cent had left education by the age of 16 years, a further $54 \%$ continued to 18 years and $10 \%$ attended higher education. Thirty-five parents (95\%) were of White British ethnicity.

Two parents who completed follow-up questionnaires had omitted to complete the baseline TOPSE.

There were no differences in scores on measures of parenting self-efficacy, parenting stress or SDQ according to any demographic variables.

\section{Baseline data}

The total PSI pre-programme score was above the 90th percentile $(M=91.17, \mathrm{SD}=24.36)$, indicating clinically significant levels of stress for some parents. The total SDQ score $(M=13.32$, $\mathrm{SD}=6.77)$ fell between normal (0-13) and borderline (14-16), suggesting that some parents rated their child's behaviour as problematic. The subscale for conduct $(M=3.57, \mathrm{SD}=2.16)$ falls between borderline and abnormal, and hyperactivity $(M=5.38, \mathrm{SD}=2.93)$ falls between the normal range and borderline. Baseline scores for TOPSE were similar to those found in previous studies; emotion $(M=49.78, \quad \mathrm{SD}=8.57)$, play $(M=45.26, \mathrm{SD}=10.34)$, empathy $(M=43.16, \mathrm{SD}=$ $9.84)$, control $(M=28.55, \mathrm{SD}=10.54)$, boundaries $(M=33.35, \quad \mathrm{SD}=10.30), \quad$ pressures $\quad(M=36.63$, $\mathrm{SD}=11.67)$, acceptance $(M=44.03, \mathrm{SD}=10.59)$, learning $(M=49.36, \mathrm{SD}=7.69)$.

\section{Non-responders to follow-up}

To determine whether there were differences between parents who completed follow-up questionnaires and those who dropped out of the study, baseline scores were analysed. There were no significant differences in baseline measures of TOPSE, PSI or SDQ between participants who completed both sets of questionnaires and those who completed only baseline questionnaires. Neither were there any differences at baseline according to any demographic variables. Independent samples $t$-tests were conducted on total scale scores and subscale scores for each of the three measures.

\section{Pre- and post-programme change in scores}

There was a statistically significant increase in mean scores from baseline to follow-up on all TOPSE scales except the scale to measure learning (Table 2). There was a statistically significant difference in mean scores from baseline to follow-up on all PSI scales (Table 3). There was no significant difference in SDQ scores except for the scale to measure conduct (Table 4).

\section{Correlations}

A Pearson's correlation coefficient was computed to assess the relationship between TOPSE and PSI pre-programme and at three-month followup. There was a positive correlation between the 
Table 2 Mean change in scores for TOPSE

\begin{tabular}{|c|c|c|}
\hline \multirow[t]{2}{*}{ TOPSE } & $n=35$ & \multirow[t]{2}{*}{$P$} \\
\hline & Mean $(95 \% \mathrm{Cl})$ & \\
\hline Emotion \& Affection & $3.5(1.4,5.6)$ & 0.002 \\
\hline Play & $5.3(1.5,8.3)$ & 0.001 \\
\hline Empathy \& Understanding & $6.5(3.7,9.4)$ & 0.000 \\
\hline Control & $10.3(6.8,13.8)$ & 0.000 \\
\hline Discipline \& Boundaries & $9.2(6.0,12.3)$ & 0.000 \\
\hline Pressures & $4.7(0.7,8.8)$ & 0.023 \\
\hline Self-acceptance & $4.5(1.8,7.3)$ & 0.002 \\
\hline Learning \& Knowledge & $2.5(-0.5,5.4)$ & 0.100 \\
\hline Total & $46.5(28.1,65.0)$ & 0.000 \\
\hline
\end{tabular}

TOPSE $=$ tool to measure parenting self-efficacy.

Table 3 Mean change in scores for PSI

\begin{tabular}{lll}
\hline PSI & $n=37$ & $P$ \\
\cline { 2 - 2 } & Mean $(95 \% \mathrm{Cl})$ & \\
\hline Parental distress & $2.4(4.5,0.3)$ & 0.024 \\
Parent-child & $1.5(3.0,0.0)$ & 0.050 \\
dysfunctional interaction & & \\
Difficult child & $4.6(7.0,2.2)$ & 0.000 \\
Total difficulties & $8.5(13.5,3.5)$ & 0.002 \\
\hline
\end{tabular}

$\mathrm{PSI}=$ parenting stress index .

Table 4 Mean change in scores for SDO

\begin{tabular}{lcl}
\hline SDO & \multicolumn{1}{l}{$n=37$} & $P$ \\
\cline { 2 - 2 } & \multicolumn{1}{l}{ Mean $(95 \% \mathrm{Cl})$} & \\
\hline Emotion & $0.42(1.0,-0.2)$ & 0.180 \\
Conduct & $0.74(1.2,0.3)$ & 0.004 \\
Hyperactivity & $0.24(0.9,-0.4)$ & 0.462 \\
Peer problems & $-0.11(0.3,-0.5)$ & 0.629 \\
Prosocial & $-0.42(0.2,-1.1)$ & 0.192 \\
Total SDQ score & $1.29(2.7,-0.1)$ & 0.063 \\
\hline
\end{tabular}

$\mathrm{SDQ}=$ strength and difficulties questionnaire.

two variables pre-programme $(r=774, n=58$, $P<.001)$ and at three-month follow-up $(r=715$, $n=37, P<.001)$. Scatterplots summarise the results (Figures 1 and 2). A Pearson's correlation coefficient was also computed to assess the relationship between TOPSE and SDQ pre-programme and at three-month follow-up. A weak correlation was found pre-programme $(r=445, \quad n=58, P<.001)$ and at three-month follow-up $(r=.016, n=37$, $P=.001)$.

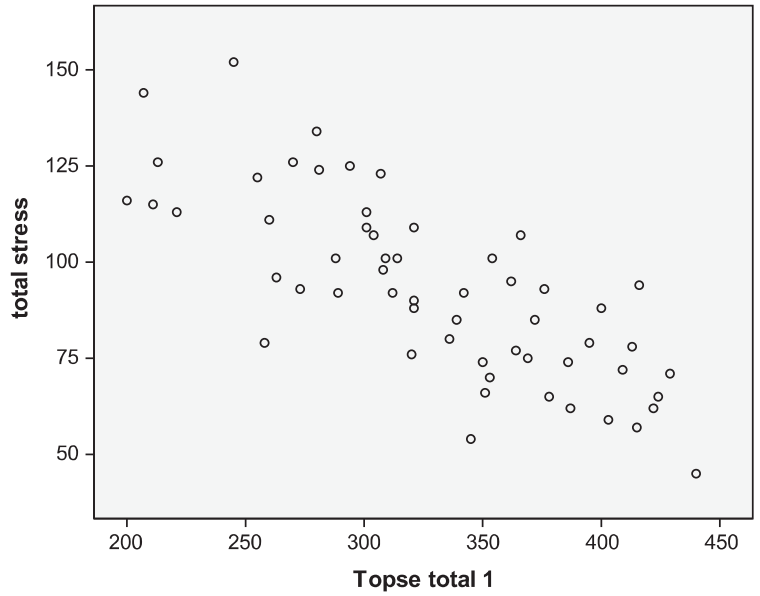

Figure 1 Pre-programme

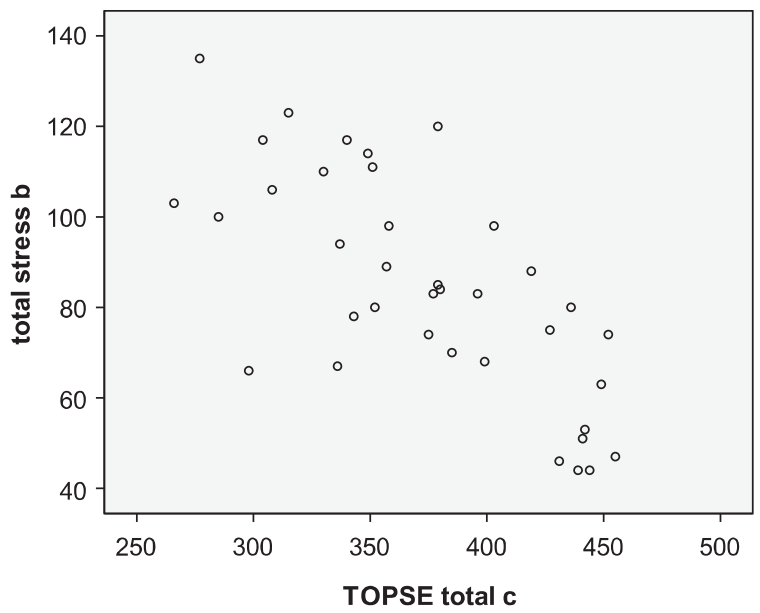

Figure 2 Three-month follow-up

\section{Discussion}

Parents demonstrated a significant change in both parenting self-efficacy and parenting stress after attending a parenting programme. At the start of the programme, many parents reported significantly high levels of parenting stress and these scores were within the normal range three months after attending the parenting programme. Parenting self-efficacy also improved three months following the programme, consistent with previous studies (Bloomfield and Kendall, 2007; 2010). The correlations between 
parenting stress and parenting self-efficacy showed that as self-efficacy increased, levels of parenting stress decreased. This clearly suggests that parents who are feeling less confident in their parenting are also experiencing higher levels of stress and that greater confidence is related to less stress. This was found both at baseline measures and at threemonth follow-up. The tools used to measure parenting self-efficacy and parenting stress have no overlapping items.

These findings are consistent with other studies that demonstrate that parenting stress and parenting self-efficacy co-vary (Jones and Prinz, 2005; Sevigny and Loutzenhiser, 2009). Although we cannot infer a causal relationship in the current study, the results support the research evidence that parental perceptions of competence determine feelings of stress (Mash and Johnston, 1990; Vondra and Belsky, 1993). The interaction between parenting stress and parenting self-efficacy has also been found to be a significant predictor of paediatric primary care use in the United States (Janicke and Finney, 2003). The finding also supports Belskey's earlier work on determinants of parenting that psychosocial support is a key variable (Belsky, 1984). This may suggest implications for parenting confidence and appropriate use of health-care services for children in the United Kingdom and is worthy of further research.

There was no significant change from the start of the programme to three-month follow-up on any measure of child strengths and difficulties except for the scale to measure conduct. The reasons given for attending parenting programmes are often around coping with child conduct (Bloomfield et al., 2005) and this was reflected in the baseline SDQ scores. The mean baseline scores for the scale to measure conduct fell between the borderline and clinical range. Although there was an improvement in conduct at three months, the mean scores remained in the borderline range. A number of studies have found improvements in child conduct scores post programme, but these have not been maintained at 12 months and beyond (Patterson et al., 2002; Anderson et al., 2005) and no difference was found between control group and intervention group on any measure of child emotional or behavioural adjustment at 12 months (Stewart-Brown et al., 2004). Studies that have found post-programme improvements in child behaviour have tended to focus on samples with a high incidence of challenging behaviour than the general population (Bywater et al., 2011).

We did not find child behaviour to be strongly associated with parenting self-efficacy and this supports the finding that child difficultness does not emerge as a predictor of parenting selfefficacy (Sevigny and Loutzenhiser, 2009). Parenting programmes are designed to support parents and to facilitate and empower them in their parenting role. Opportunities are provided to enhance parent's self-belief that they are more able to cope with their child's difficult behaviour. Many parents seem to gain a fairly rapid insight when attending programmes that it is their own behaviours and response to their child that needs to change before changes in child behaviour can take place. Perhaps increased parental confidence and lower parental stress are necessary precursors to changes in child behaviour. Changes in the child may occur over a longer period once both parent and child have adjusted to new parenting techniques and new ways of interacting with each other. This study adds to the evidence that parent outcomes may be a more reliable and appropriate measure of programme effectiveness than child outcomes at least in the short term. The association between parenting selfefficacy and parenting stress indicates that both TOPSE and the PSI may be useful predictive indicators of positive parenting that may in the longer term have implications for child behaviour. This needs further testing.

The Healthy Child Programme (Department of Health, 2009) and the current independent enquiry on early intervention evidence (Allen, 2011) focus on the need to use reliable evidence to develop parenting interventions that will support child development in the early years. The evidence from this study also contributes to the current emphasis in the Department of Health for England on developing the health visiting service (Department of Health, 2011). Although the facilitators in this study were not all health visitors, the implications for expanding their role with parents in line with current Department of Health policy is worthy of further consideration.

\section{Limitations and conclusions}

There are some limitations of the study. The study was undertaken with a small sample of parents attending one type of parenting programme. Further 
research over a longer period and with a larger sample to explore the effects of a range of parenting programmes may yield other findings and provide further generalisable evidence for parent and child outcomes. As with many before and after studies, there was an inevitable loss to follow-up. However, no differences were found in baseline scores between parents who dropped out of the study and those who completed the follow-up questionnaires; therefore, we feel the results were not confounded in terms of selection bias. Neither were differences found in outcome measures dependent on any demographic variables. Owing to the correlation nature of the study, it has not been possible to make any causal inferences between parenting stress and parenting self-efficacy. Furthermore, we have made no correction for facilitator style, which may confound the findings. Further research that takes account of facilitator style would also be of interest. This study has nonetheless provided evidence to suggest a relationship between parenting selfefficacy and parenting stress; parents who feel less efficacious experience higher levels of stress, whereas greater parenting self-efficacy is associated with lower stress. Parent outcomes may be a more reliable measure of programme effectiveness than child outcomes at least in the short term.

\section{Acknowledgements}

We thank all participating parents and parenting programme facilitators for their help and support.

\section{References}

Abidin, R. and Burke, W. 1978: The development of the parenting stress index. Annual meeting of the American Psychological Association. Toronto: American Psychological Association.

Abidin, R.R. 1986: Parenting stress index. Charlottesville, VA. Pediatric Psychology Press.

Abidin, R.R. 1997: Parenting stress index: a measure of the parent child system. In Zalaquett, C. and Woods, R., editors, Evaluating stress: a book of resources (pp. 277-291). Lanham MD: Scarecrow Press Inc.

Allen, G. 2011: Early intervention: the next steps. London: HMG.

Anderson, L., Vostanis, P. and O'reilly, M. 2005: Three-year follow-up of a family support service cohort of children with behavioural problems and their parents. Child, care, health and development 31, 469-77.

Bandura, A. 1982: Self-efficacy mechanism in human agency. American Psychologist 37, 122-47.
Bandura, A. 1986: Social foundations of thought and action: a social cognitive theory. Engle Wood Cliffs, NJ: Prentice Hall.

Bandura, A. 1989: Regulation of cognitive processes through perceived self-efficacy. Developmental Psychology 25, 729-35.

Bandura, A. 1997: Self-efficacy: the exercise of control. New York: Freeman.

Barlow, J. and Stewart-Brown, S. 2000: Behaviour problems and group-based parent education programmes. Developmental and Behavioral Pediatrics 21, 356-70.

Belsky, J. 1984: The determinants of parenting: a process model. Child Development 55, 83-96.

Bloomfield, L. and Kendall, S. 2007: Testing a parenting programme evaluation tool as a pre- and post-course measure of parenting self-efficacy. Journal of Advanced Nursing 60, 487-93.

Bloomfield, L. and Kendall, S. 2010: Audit as evidence: the effectiveness of '123 Magic' programmes. Community Practitioner 83, 26-30.

Bloomfield, L., Kendall, S., Applin, L., Attarzadeh, V., Dearnley, K., Edwards, L., Hinshelwood, L., Lloyd, P. and Newcombe, T. 2005: A qualitative study exploring the experiences and views of parents, health visitors and family support centre workers on the challenges and difficulties of parenting. Health and Social Care in the Community 13, 46-55.

Bradley, S.J., Jadaa, D., Brody, J., Landy, S., Tallett, S., Watson, W., Shea, N. and Stephens, D. 2003: Brief psychoeducational parenting program: an evaluation and 1-year follow-up. Journal of the American Academy of Child and Adolescent Psychiatry 42, 1171-78.

Bywater, T., Hutchings, J., Linck, P., Whitaker, C., Daley, D., Yeo, S.T. and Edwards, R.T. 2011: Incredible years parent training support for foster carers in Wales: a multi-centre feasibility study. Child, Care, Health and Development 37(2), 233-243.

CEBC California Evidence-Based Clearinghouse for Child Welfare, 2006. www.cebc4cw.org.

Coleman, P. and Karraker, K. 1998: Self-efficacy and parenting quality: findings and future applications. Developmental Review 18, 47-85.

Coleman, P. and Karraker, K. 2000: Parenting self-efficacy among mothers of school-age children: conceptualization, measurement and correlates. Family Relations 49, 13-24.

Coleman, P.K. and Karraker, K.H. 2003: Maternal self-efficacy beliefs, competence in parenting, and toddlers behaviour and development status. Infant Mental Health Journal 24, $126-48$

Deater-Deckard, K. 1998: Parenting stress and child adjustment: some old hypotheses and new questions. Clinical Psychology: Science and Practice 5, 314-32.

Deater-Deckard, K. and Scarr, S. 1996: Parenting stress among dual-earner mothers and fathers: are there gender differences? Journal of Family Psychology 10, 45-59.

Department of Health. 2009: The healthy child programme. London: Department of Health. 
Department of Health. 2011: The health visiting implementation plan. London: Department of Health.

Edwards, R.T., Ceilleachair, A., Bywater, T., Hughes, D. and Hutchings, J. 2007: Parenting programmes for parents of children at risk of developing conduct disorder: cost effectiveness analysis. British Medical Journal 334, 682-85.

Gardner, F., Burton, J. and Klimes, I. 2006: Randomised controlled trial of a parenting intervention in the voluntary sector for reducing child conduct problems: outcomes and mechanisms of change. Journal of Child Psychology and Psychiatry 47, 1123-32.

Goodman, R. 1997: The strengths and difficulties questionnaire: a research note. Journal of Child Psychology and Psychiatry 38, 581-86.

Goodman, S.H. and Gotlib, I.H. 2002: Children of depressed parents: alternative pathways to risk for psychopathology. Washington, DC: American Psychological Association Press.

Gross, D. and Tucker, S. 1994: Parenting confidence during toddlerhood: a comparison of mothers and fathers. Nurse Practitioner 19, 19-29.

Her Majesty's Government 2006: Reaching out: an action plan on social exclusion. London: Cabinet Office.

Janicke, D.M. and Finney, J.W. 2003: Children's primary health care services: social-cognitive factors related to utilization. Journal of Paediatric Psychology 28, 547-57.

Jones, T. and Prinz, R. 2005: Potential roles of parental selfefficacy in parent and child adjustment: a review. Clinical Psychology Review 25, 341-63.

Kendall, S. 1991: A home visit by a health visitor using Bandura's theory of self-efficacy. In While, A., editor, Caring for children: towards partnership with families. London: Arnold.

Kendall, S. and Bloomfield, L. 2005: Developing and validating a tool to measure parenting self-efficacy. Journal of Advanced Nursing 51, 174-81.

Macdonald, G. 2001: Effective interventions for child abuse and neglect. An evidence-based approach to planning and evaluating interventions. Chichester: John Wiley and Sons Ltd.

Mash, E. and Johnston, C. 1990: Determinants of parenting stress: illustrations from families of hyperactive children and families of physically abused children. Journal of Clinical Child Psychology 19, 313-28.

Ostberg, M. and Hagekull, B. 2000: A structural modeling approach to the understanding of parenting stress. Journal of Clinical Child Psychology 29, 615-25.

Patterson, J., Barlow, J., Mockford, C., Klimes, I., Pyper, C. and Stewart-Brown, S. 2002: Improving mental health through parenting programmes: block randomised controlled trial. Archives of Disease in Childhood 87, 472-77.

Petrie, J., Bunn, F. and Byrne, G. 2007: Parenting programmes for preventing tobacco, alcohol or drugs misuse in children $<18$ : a systematic review. Health Education Research 37(2), 233-243.

Phelan, T. 2004: 1-2-3 magic: effective discipline for children. Child Management Inc., Illinois.

Putnam, S.P., Sanson, A.V. and Rothbar, M.K. 2002: Child temperament and parenting. In Bornstein, M.H., editor, Handbook of parenting, second edition. Mahwah, NJ: Erlbaum.

Raikes, H. and Thompson, R. 2005: Efficacy and social support as predictors of parenting stress among families in poverty. Mental Health Journal 26, 177-90.

Scott, M.J. and Stradling, S. 1987: Evaluation of a group program for parents of problem children. Behavioural Psychotherapy 15, 224-39.

Scott, S. 1998: Intensive interventions to improve parenting. Archives of Disease in Childhood 79, 90-93.

Scott, S., Knapp, M., Henderson, J. and Maughn, B. 2001a: Financial cost of social exclusion: follow up study of antisocial children into childhood. British Medical Journal 323, 1-5.

Scott, S., Spender, Q., Doolan, M., Jacobs, B. and Aspland, H. 2001b: Multicentre controlled trial of parenting groups for childhood antisocial behaviour in clinical practice. British Medical Journal 323, 194-97.

Sevigny, P.R. and Loutzenhiser, L. 2009: Predictors of parenting self-efficacy in mothers and fathers of toddlers. Child: care, health and development 36, 179-98.

Stewart-Brown, S. 2008: Improving parenting: the why and the how. Archives Of Disease In Childhood 93, 102-104.

Stewart-Brown, S., Paterson, J., Mockford, C., Barlow, J., Klimes, I. and Pyper, C. 2004: Impact of a general practice based group parenting programme: quantitative and qualitative results from a controlled trial at 12 months. Archives of Disease in Childhood 89, 519-25.

Teti, D. and Gelfand, D. 1991: Behavioural competence among mothers of infants in the first year: The mediational role of maternal self-efficacy. Child Development 62, 918-29.

Vondra, J. and Belsky, J. 1993: Developmental origins of parenting: personality and relationship factors. In Luster, T. and Okagaki, L., editors, Parenting: an ecological perspective. Hillsdale, NJ: Erlbaum.

World Health Organization. 2002: A review: a critical link, intervention for physical growth and development. Child and adolescent health and development. Geneva: World Health Organisation. 